\title{
Hitting Simplices with Points in $\mathbb{R}^{3}$
}

\author{
Abdul Basit · Nabil H. Mustafa • Saurabh Ray • \\ Sarfraz Raza
}

Received: 30 January 2009 / Revised: 7 February 2010 / Accepted: 9 May 2010 /

Published online: 28 May 2010

(C) Springer Science+Business Media, LLC 2010

\begin{abstract}
The so-called first selection lemma states the following: given any set $P$ of $n$ points in $\mathbb{R}^{d}$, there exists a point in $\mathbb{R}^{d}$ contained in at least $c_{d} n^{d+1}-O\left(n^{d}\right)$ simplices spanned by $P$, where the constant $c_{d}$ depends on $d$. We present improved bounds on the first selection lemma in $\mathbb{R}^{3}$. In particular, we prove that $c_{3} \geq 0.00227$, improving the previous best result of $c_{3} \geq 0.00162$ by Wagner (On $k$-sets and applications. Ph.D. thesis, ETH Zurich, 2003). This makes progress, for the three-dimensional case, on the open problems of Bukh et al. (Stabbing simplices by points and flats. Discrete Comput. Geom., 2010) (where it is proven that $c_{3} \leq 1 / 4^{4} \approx 0.00390$ ) and Boros and Füredi (The number of triangles covering the center of an $n$-set. Geom. Dedic. 17(1):69-77, 1984) (where the two-dimensional case was settled).
\end{abstract}

Keywords Centerpoint $\cdot$ Selection lemma $\cdot$ Simplex

\section{Introduction}

The First Selection Lemma (see Chap. 9 in [7]) states the following: given any set $P$ of $n$ points in $\mathbb{R}^{d}$, there exists a point in $\mathbb{R}^{d}$ contained in at least $c_{d} n^{d+1}-O\left(n^{d}\right)$ sim-

\footnotetext{
A. Basit · N.H. Mustafa ( $\varangle) \cdot$ S. Raza

Department of Computer Science, LUMS, Lahore, Pakistan e-mail: nabil@1ums.edu.pk
}
A. Basit
e-mail: abdulbasit@lums.edu.pk
S. Raza
e-mail: sarfrazr@lums.edu.pk

S. Ray

Mathematics Department, EPFL, Lausanne, Switzerland

e-mail: saurabh@epfl.ch 
plices spanned by $P$. It is a fundamental result that has had several applications in discrete geometry [7]. In particular, it was used to construct weak $\epsilon$-nets [2], which were crucially used in the solution of the famous Hadwiger-Debrunner conjecture [1].

The first selection lemma is closely linked to the following useful property of point sets: given any set $P$ of $n$ points in $\mathbb{R}^{d}$, there always exists a point $q$ such that any closed halfspace containing $q$ contains at least $n /(d+1)$ points of $P$. Such a point is called a centerpoint. In general, a point $p$ has depth $t$ w.r.t. $P$ if any closed halfspace containing $p$ contains at least $t$ points of $P$. The centerpoint theorem guarantees a point of depth $n /(d+1)$. The depth of $P$ is defined as the maximum depth, w.r.t. $P$, of any point $p \in \mathbb{R}^{d}$. Currently the best known bounds for $c_{d}$ for $d \geq 3$ are achieved by using an arbitrary centerpoint.

In the past 27 years, the value of the constant $c_{d}$ has been investigated in a series of papers. Unfortunately, there still remains a large gap between the current upper and lower bounds. Bárány [3] proved that $c_{d} \geq \frac{1}{d !(d+1)^{d+1}}$. For $d=2$, this proves the existence of a point in $n^{3} / 54$ of the triangles spanned by any point set of size $n$ in the plane. This was improved to $n^{3} / 27$ in [4] (see Bukh [5] for another proof, which is simple and elegant) and was later shown to be optimal in [6]. Bárány's bound was improved to $\frac{d^{2}+1}{(d+1) !(d+1)^{d+1}}$ by Wagner [8], who in fact showed that any point of depth $\tau n$ is contained in at least the following number of simplices:

$$
\frac{(d+1) \tau^{d}-2 d \tau^{d+1}}{(d+1) !} n^{d+1}-O\left(n^{d}\right) .
$$

The recent work of Bukh, Matoušek, and Nivasch [6] is devoted to the investigation of upper bounds on the value of $c_{d}$. In particular, they show that in $\mathbb{R}^{d}$, taking an arbitrary centerpoint cannot give a better bound than the one above (set $\tau=1 /(d+1)$ ). Their main result is an elegant construction of a point set $P$ such that no point in $\mathbb{R}^{d}$ is contained in more than $(n /(d+1))^{d+1}$ simplices spanned by $P$. Furthermore, they conjecture that this is the right bound and leave improving the lower bound as the main open problem.

Our Result We make progress on the above problem, which was left as the main open problem in [6], by improving the bounds for the first selection lemma in $\mathbb{R}^{3}$ by a factor of 1.4. The previous best result, which follows from (1), proves that given any $P$, there exists a point contained in at least $0.00162 n^{4}$ simplices (this can be any centerpoint). Our main result, presented in Sect. 2, is the following:

Theorem 1.1 Let $P$ be a set of $n$ points in $\mathbb{R}^{3}$. Then there exists a point contained in at least $0.00227 n^{4}$ simplices spanned by $P$.

While our result does not settle the question, it shows the current lower bounds that are not tight and gives more strength to their conjecture. The technique we use can, in principle, be generalized to higher dimensions. However, there are some technical difficulties in proving a nontrivial lower bound for the function defined in Lemma 2.3. We solely concentrate on the three-dimensional problem since it is the smallest dimension in which the value of $c_{d}$ is not known exactly. 


\section{Improving First Selection Lemma in $\mathbb{R}^{3}$}

The bound of Wagner [8] improves with the depth of the point set $P$. Our simple idea is to show that when the depth of $P$ is low, one can also get a better bound. For example, when $\operatorname{depth}(P)=n /(d+1)$, then the conjecture of [6] is in fact proven below. By combining the two approaches, one gets an overall improvement.

We will use the following lemma, which follows easily from a lemma proved in [4].

Lemma 2.1 Given a set $P$ of $n$ points in $\mathbb{R}^{d}$, where $\operatorname{depth}(P)=\tau n-1$, there exist a point $p$ with depth $\tau n-1$ and a set $\mathcal{H}$ of $d+1$ open halfspaces $\left\{h_{1}, \ldots, h_{d+1}\right\}$ such that (i) $\left|h_{i} \cap P\right|=\tau n$, (ii) $p$ lies on the boundary plane of each $h_{i}$, and (iii) $h_{1} \cup \ldots \cup$ $h_{d+1}$ cover the entire $\mathbb{R}^{d}$ except the point $p$.

Proof Boros and Füredi [4] (Lemma 3) prove that given a point set $P$ of size $n$ and depth $\sigma$ in $\mathbb{R}^{d}$, there exist a point $p$ of depth $\sigma$ and $d+1$ closed halfspaces $\eta_{1}, \ldots, \eta_{d+1}$ which cover $\mathbb{R}^{d}$, have the point $p$ on their boundary, and where $\left|\eta_{i} \cap P\right|=n-\sigma-1$ (they actually prove this statement for $\mathbb{R}^{2}$, but as they also note in their paper, the generalization to $\mathbb{R}^{d}$ is straightforward). Applying this lemma with $\sigma=\tau n-1$ and setting $h_{i}$ to be the complement of $\eta_{i}$ for $i=1, \ldots, d+1$ proves the required statement.

We now prove a technical lemma which can be seen as a generalization of Carathéodory's theorem. Given a set $P$ of points in $\mathbb{R}^{d}, \operatorname{conv}(P)$ denotes the convex hull of $P$.

Lemma 2.2 Let $P=\left\{p_{1}, \ldots, p_{d+2}\right\}$ be a set of $d+2$ points in $\mathbb{R}^{d}$. Then any point $x \in \operatorname{conv}(P)$ lies in at least two $d$-simplices spanned by $P$.

Proof If $x$ lies on any facet $F$ of $\operatorname{conv}(P)$, then the (at least) two simplices spanned by $P$ that contain $F$ also contain $x$. Otherwise take any point of $P$, say $p_{1}$, and consider the ray emanating from $p_{1}$ and passing through $x$. This ray, after passing through $x$, intersects the boundary of $\operatorname{conv}(P)$ in a $(d-1)$-simplex spanned by $d$ points, say $P^{\prime}$. Then $P^{\prime} \cup p_{1}$ contains $x$ and has size $d+1$. Let $p_{i}$ be the remaining point in $P \backslash\left(P^{\prime} \cup\left\{p_{1}\right\}\right)$. Repeating the same procedure of shooting a ray from $p_{i}$ through $x$ results in another $d$-simplex, with $p_{i}$ as one of its points, that contains $x$.

Given a set $P$ of $n$ points in $\mathbb{R}^{3}$, with $\operatorname{depth}(P)=\tau n-1$, use Lemma 2.1 to get the point $p$ and a set of four halfspaces $\mathcal{H}=\left\{h_{1}, h_{2}, h_{3}, h_{4}\right\}$ satisfying the stated conditions. The rest of the paper will be devoted to proving that $p$ is contained in a lot of simplices spanned by $P$ (w.l.o.g. one can assume that $p \notin P$, otherwise the bound can only improve, as all the $\Theta\left(n^{d}\right)$ simplices defined by $p$ contain $p$ ). For any halfspace $h$, let $\bar{h}$ be the complement halfspace of $h$, and $\partial h$ be its boundary plane. 
Define the following subsets of $P$ for all $i, j=1, \ldots, 4$ :

$$
\begin{aligned}
A_{i} & =P \cap\left(\bigcap_{l \neq i} \bar{h}_{l}\right) \cap h_{i}, \quad B_{i, j}=P \cap\left(\bigcap_{l \neq i, j} \bar{h}_{l}\right) \cap h_{i} \cap h_{j}, \\
C_{i} & =P \cap\left(\bigcap_{l \neq i} h_{l}\right) \cap \bar{h}_{i} .
\end{aligned}
$$

Set $\alpha_{i}=\left|A_{i}\right| / n, \beta_{i, j}=\left|B_{i, j}\right| / n$, and $\gamma_{i}=\left|C_{i}\right| / n$. Our main lemma is the following.

Lemma 2.3 Let $P$ be a set of $n$ points in $\mathbb{R}^{3}$, with depth $(P)=\tau n-1$. Then, there exists a point contained in at least $g(P) \cdot n^{4}$ simplices spanned by $P$, where

$$
g(P)=\left(\prod_{i} \alpha_{i}\right)+\left(\sum_{i<j} \beta_{i, j} \frac{\prod_{l} \alpha_{l}}{\max \left(\alpha_{i}, \alpha_{j}\right)}\right)+\left(\sum_{i} \gamma_{i} \frac{\prod_{l} \alpha_{l}}{\max _{l \neq i} \alpha_{l}}\right) .
$$

Proof Let $p$ be the point from Lemma 2.1, together with the four halfspaces $h_{1}, \ldots, h_{4}$. We first show that the simplex spanned by any four points, one from each of $A_{i}$, will always contain $p$.

Claim 2.4 Let $p_{1}, p_{2}, p_{3}, p_{4} \in P$ be four points of $P$ such that $p_{i} \in A_{i}$. Then the simplex spanned by these four points contains $p$.

Proof Assume for contradiction that conv $\left(\left\{p_{1}, p_{2}, p_{3}, p_{4}\right\}\right)$ does not contain $p$. Then there exists a hyperplane $h$ that separates $p$ from $\operatorname{conv}\left(\left\{p_{1}, p_{2}, p_{3}, p_{4}\right\}\right)$ and does not contain $p$. For $i \in\{1,2,3,4\}$, define $q_{i}$ to be the point $p p_{i} \cap h$. By definition, each $h_{i}$ passes through $p$, contains $p_{i}$ and does not contain any other point $p_{j}$ with $j \neq i$. Note that then each $h_{i}$ also contains $q_{i}$. Moreover, $h_{i}$ does not contain $q_{j}$ with $i \neq j$ because $h_{i}$ does not contain $p_{j}$ and has $p$ only on its boundary. By Radon's theorem [7, p. 9] applied to $\left\{q_{1}, q_{2}, q_{3}, q_{4}\right\}$ lying on the plane $h$, there exist disjoint sets $Q_{1}, Q_{2}$ and a point $s$ such that $Q_{1} \cup Q_{2}=\left\{q_{1}, q_{2}, q_{3}, q_{4}\right\}$ and $s \in \operatorname{conv}\left(Q_{1}\right) \cap$ $\operatorname{conv}\left(Q_{2}\right)$. Since $s$ lies on $h, s \neq p$. By convexity, any halfspace that contains $s$ must also contain at least one point from both $Q_{1}$ and $Q_{2}$. As $\mathcal{H}$ covers $\mathbb{R}^{3} \backslash\{p\}$, there exists an $i$ such that $s \in h_{i}$. But this gives a contradiction, as then this $h_{i}$ must contain at least one point from both $Q_{1}$ and $Q_{2}$, and so contain some point $q_{j}$ with $j \neq i$.

The total number of such simplices is $n^{4} \cdot \prod_{i} \alpha_{i}$, which is the first term in (3). Call any such simplex a basic simplex, i.e., a simplex on $p_{1}, p_{2}, p_{3}, p_{4} \in P$ is basic iff $p_{i} \in A_{i}$ for all $i$. All other simplices are called nonbasic.

Now we use basic simplices, which always contain $p$, to prove the existence of several other simplices which must also contain $p$.

Claim 2.5 Let $P^{\prime}=\left\{p_{1}, p_{2}, p_{3}, p_{4}, p_{5}\right\} \subset P$ be five points of $P$ such that $p_{k} \in A_{k}$, $k=1, \ldots, 4$, and $p_{5} \in B_{i, j}$ for any $1 \leq i<j \leq 4$. Then the simplex spanned either by $P^{\prime} \backslash p_{i}$ or by $P^{\prime} \backslash p_{j}$ contains $p$. 
Proof By Claim 2.4, the basic simplex spanned by $p_{1}, p_{2}, p_{3}, p_{4}$ contains $p$. Therefore, by Lemma 2.2, at least one other simplex spanned by $P^{\prime}$ must contain $p$. Note that this simplex must have $p_{5}$ as one of its points. Also, it must contain $p_{k}$, where $k \neq i, j$, since the plane $\partial h_{k}$ separates $P^{\prime} \backslash p_{k}$ from $p$, as from the definitions (2) it follows that $p_{l} \in \bar{h}_{k}$ for all $l \neq k$ and $p_{5} \in \bar{h}_{k}$ since $p_{5} \in B_{i, j}$. So for this second simplex, the only possible choice is for the fourth vertex, which can be either $p_{i}$ or $p_{j}$.

For any fixed $i, j$, there are $n^{5}\left(\beta_{i, j} \prod_{i} \alpha_{i}\right) 5$-tuples as in Claim 2.5, and each produces one $d$-simplex containing $p$. Each such $d$-simplex may be double-counted at most $n \max \left(\alpha_{i}, \alpha_{j}\right)$ times, so the total number of distinct $d$-simplices of the type in Claim 2.5 containing $p$ is at least $n^{4} \frac{\beta_{i, j} \prod_{i} \alpha_{i}}{\max \left(\alpha_{i}, \alpha_{j}\right)}$, which when summed over all $i<j$, forms the second term in (3).

Claim 2.6 Let $P^{\prime}=\left\{p_{1}, p_{2}, p_{3}, p_{4}, p_{5}\right\} \subset P$ be five points of $P$ such that $p_{k} \in A_{k}$, $k=1, \ldots, 4$, and $p_{5} \in C_{i}$ for any $1 \leq i \leq 4$. Then there $i$ a two-element subset $P^{\prime \prime} \subset P^{\prime} \backslash\left\{p_{5}, p_{i}\right\}$ such that the simplex conv $\left(\left\{p_{5}, p_{i}\right\} \cup P^{\prime \prime}\right)$ contains $p$.

Proof As in Claim 2.5, at least one nonbasic simplex spanned by $P^{\prime}$ must contain $p$, with $p_{5}$ as one of its points. Also, it must contain $p_{i}$ : the plane $\partial h_{i}$ separates $P^{\prime} \backslash p_{i}$ from $p$, as $P^{\prime} \backslash p_{i} \subseteq \bar{h}_{i}$. The other two vertices of this second simplex must therefore be a subset of the remaining three vertices in $P^{\prime}$.

By similarly eliminating the double-counting, the $d$-simplices from Claim 2.6 form the third term of $g(P)$. Finally, note that no two simplices are counted twice in $g(P)$, since each contains exactly one point from a distinct region (one of $B_{i, j}$ or $C_{i}$ ).

Note that we only have these two constraints on the nonnegative variables $\alpha_{i}, \beta_{i, j}$, and $\gamma_{i}$ :

$$
\begin{aligned}
& \tau=\frac{\left|h_{i} \cap P\right|}{n}=\alpha_{i}+\sum_{j \neq i} \beta_{i, j}+\sum_{j \neq i} \gamma_{j} \quad \text { for each } i=1, \ldots, 4 \\
& \sum_{i} \alpha_{i}+\sum_{i<j} \beta_{i, j}+\sum_{i} \gamma_{i}=1, \quad \text { as } \mathcal{H} \text { covers } \mathbb{R}^{3} \backslash\{p\} \text { and } p \notin P .
\end{aligned}
$$

It remains to show that regardless of the distribution of the points in the disjoint sets $A_{i}, B_{i, j}$, and $C_{j}$, and therefore the values of the variables satisfying (4) and (5), the quantity $g(P)$ is always bounded suitably from below.

Lemma 2.7 Let $P$ be a set of $n$ points in $\mathbb{R}^{3}$, with $\operatorname{depth}(P)=\tau n-1$, and $g(P)$ as in Lemma 2.3. If $\tau \leq 0.3$, then $g(P) \geq \tau(1-3 \tau)^{2}(5 \tau-1)$. 
Proof Using the fact that $\alpha_{i} \leq \tau$, we get

$$
\begin{aligned}
g(P) & =\left(\prod_{i} \alpha_{i}\right)+\left(\sum_{i<j} \beta_{i, j} \frac{\prod_{l} \alpha_{l}}{\max \left(\alpha_{i}, \alpha_{j}\right)}\right)+\left(\sum_{i} \gamma_{i} \frac{\prod_{l} \alpha_{l}}{\max _{l \neq i} \alpha_{l}}\right) \\
& \geq\left(\prod_{i} \alpha_{i}\right)+\left(\sum_{i<j} \beta_{i, j} \frac{\prod_{l} \alpha_{l}}{\tau}\right)+\left(\sum_{i} \gamma_{i} \frac{\prod_{l} \alpha_{l}}{\tau}\right) \\
& =\left(\prod_{i} \alpha_{i}\right)\left(1+\frac{\sum_{i<j} \beta_{i, j}+\sum_{i} \gamma_{i}}{\tau}\right) .
\end{aligned}
$$

Summing up (4) for all four halfspaces and then subtracting (5), we get

$$
\sum_{i<j} \beta_{i, j}+2 \sum_{i} \gamma_{i}=4 \tau-1
$$

Therefore, $\sum_{i<j} \beta_{i, j}+\sum_{i} \gamma_{i} \leq 4 \tau-1$. This fact, together with (4), implies that $1-3 \tau \leq \alpha_{i} \leq \tau$ for $i=1, \ldots, 4$. Assuming that $\tau \leq 0.3$, we can show the following:

Claim 2.8 The bound in (6) subject to constraints (4) and (5) is minimized when $\sum_{i} \gamma_{i}=0$ or equivalently, when $\sum_{i<j} \beta_{i, j}+\sum_{i} \gamma_{i}=4 \tau-1$.

Proof Suppose that $\sum_{i} \gamma_{i}=\epsilon+\epsilon_{1}$, where $\gamma_{1}=\epsilon_{1}>0$. We show that the variables $\alpha_{i}, \beta_{i, j}, \gamma_{1}$ can be readjusted to new values $\alpha_{i}^{\prime}, \beta_{i, j}^{\prime}, \gamma_{1}^{\prime}$ to give a smaller value in (6), while still satisfying all the constraints in (4) and (5), and where $\gamma_{1}^{\prime}=0$. As long as $\sum_{i} \gamma_{i}>0$, we can iteratively apply this procedure for all $\gamma_{j}>0$ to make $\sum_{i} \gamma_{i}=0$ without increasing the lower bound. At each such step, a value of $\gamma_{j}>0$, for some $j$, is set to 0 , so this procedure finishes after at most 4 steps.

Set $\gamma_{1}^{\prime}=0, \beta_{i, j}^{\prime}=\beta_{i, j}+\frac{2 \epsilon_{1}}{3}$ for all $i, j \neq 1$, and $\alpha_{i}^{\prime}=\alpha_{i}-\epsilon_{1} / 3$ for all $i \neq 1$. One can verify that (4) and (5) still hold. Therefore, (7) also holds; in particular, it follows that $1-3 \tau \leq \alpha_{i}^{\prime} \leq \tau$ for each $i$, and so all new $\alpha_{i}^{\prime}$ variables are still nonnegative as $\tau \leq 0.3$. Now simple calculation shows that the function of (6) can only decrease. For completeness sake, we present the explicit computations:

$$
\left(\prod_{i} \alpha_{i}^{\prime}\right)\left(1+\frac{\sum \gamma_{i}^{\prime}+\sum \beta_{i, j}^{\prime}}{\tau}\right) \leq\left(\prod_{i} \alpha_{i}\right)\left(1+\frac{\sum \gamma_{i}+\sum \beta_{i, j}}{\tau}\right) .
$$

Note that $\sum_{i<j} \beta_{i, j}+\sum_{i} \gamma_{i}=4 \tau-1-\epsilon-\epsilon_{1}$ and $\sum_{i<j} \beta_{i, j}^{\prime}+\sum_{i} \gamma_{i}^{\prime}=4 \tau-$ $1-\epsilon$. So

$$
\begin{aligned}
\left(\prod_{i \neq 1} \alpha_{i}-\frac{\epsilon_{1}}{3}\right)\left(\frac{5 \tau-1-\epsilon}{\tau}\right) & \leq\left(\prod_{i \neq 1} \alpha_{i}\right)\left(\frac{5 \tau-1-\epsilon-\epsilon_{1}}{\tau}\right), \\
\prod_{i \neq 1}\left(1-\frac{\epsilon_{1}}{3 \alpha_{i}}\right) & \leq 1-\frac{\epsilon_{1}}{5 \tau-1-\epsilon} .
\end{aligned}
$$


Since each $\alpha_{i}, i \neq 1$, can be at most $\tau-\epsilon_{1}$ (from (4)), we have to prove

$$
\begin{aligned}
\left(1-\frac{\epsilon_{1}}{3\left(\tau-\epsilon_{1}\right)}\right)^{3} & =1-\frac{3 \epsilon_{1}}{3\left(\tau-\epsilon_{1}\right)}+3\left(\frac{\epsilon_{1}}{3\left(\tau-\epsilon_{1}\right)}\right)^{2}-\left(\frac{\epsilon_{1}}{3\left(\tau-\epsilon_{1}\right)}\right)^{3} \\
& \leq 1-\frac{\epsilon_{1}}{5 \tau-1-\epsilon}
\end{aligned}
$$

By dropping the negative cubic term and simplifying, we have to show that

$$
\begin{aligned}
& 1-\frac{3 \epsilon_{1}}{3\left(\tau-\epsilon_{1}\right)}+3\left(\frac{\epsilon_{1}}{3\left(\tau-\epsilon_{1}\right)}\right)^{2} \leq 1-\frac{\epsilon_{1}}{5 \tau-1-\epsilon}, \\
& -\frac{1}{\left(\tau-\epsilon_{1}\right)}+\frac{\epsilon_{1}}{3\left(\tau-\epsilon_{1}\right)^{2}} \leq-\frac{1}{5 \tau-1-\epsilon}, \\
& -(5 \tau-1-\epsilon)+\frac{\epsilon_{1}(5 \tau-1-\epsilon)}{3\left(\tau-\epsilon_{1}\right)} \leq-\left(\tau-\epsilon_{1}\right), \\
& 4 \tau \geq 1+\epsilon-\epsilon_{1}+\frac{\epsilon_{1}(5 \tau-1-\epsilon)}{3\left(\tau-\epsilon_{1}\right)} .
\end{aligned}
$$

Since $\sum_{i<j} \beta_{i, j}+2 \sum_{i} \gamma_{i}=4 \tau-1$, we have $\sum \gamma_{i}=\epsilon+\epsilon_{1} \leq(4 \tau-1) / 2$. So $4 \tau \geq 2 \epsilon+1$, and it remains to show that

$$
\frac{\epsilon_{1}(5 \tau-1-\epsilon)}{3\left(\tau-\epsilon_{1}\right)} \leq \epsilon_{1} \quad \text { or equivalently, } \quad 2 \tau \leq 1+\epsilon-3 \epsilon_{1} .
$$

Now one can verify that $2 \tau \leq 1-3 \epsilon_{1}$, since $\epsilon_{1} \leq(4 \tau-1) / 2$ and $\tau \leq 0.3$.

It follows from Claim 2.8 that

$$
g(P) \geq\left(\prod_{i} \alpha_{i}\right)\left(1+\frac{4 \tau-1}{\tau}\right)=\left(\prod_{i} \alpha_{i}\right)\left(\frac{5 \tau-1}{\tau}\right) .
$$

Claim 2.8, together with (5), also implies that $\sum \alpha_{i}=2-4 \tau$. As $\alpha_{i} \in[1-3 \tau, \tau]$, the term $\prod \alpha_{i}$ is minimized when, say, $\alpha_{1}=\alpha_{2}=\tau$ and $\alpha_{3}=\alpha_{4}=1-3 \tau$ (and then $\left.\beta_{3,4}=4 \tau-1\right)$.

Claim 2.9 $\prod \alpha_{i}$ is minimized when $\alpha_{1}=\alpha_{2}=\tau$ and $\alpha_{3}=\alpha_{4}=(1-3 \tau)$.

Proof Recall that each $\alpha_{i}$ lies in the closed interval $[(1-3 \tau), \tau]$. If each $\alpha_{i}>(1-$ $3 \tau$ ), pick the smallest of them, say $\alpha_{4}$, set $\alpha_{4}^{\prime}=\alpha_{4}-\epsilon$ for a small enough $\epsilon>0$, and add this excess to any other variable that is less than $\tau$, say $\alpha_{3}$ (there always exists another variable less than $\tau$, else $\sum \alpha_{i}>(1-3 \tau)+3 \tau=1$, a contradiction). Then $\left(\alpha_{3}+\epsilon\right)\left(\alpha_{4}-\epsilon\right)<\alpha_{3} \alpha_{4}$ since $\alpha_{4} \leq \alpha_{3}$, minimizing the product further. Similarly, $\alpha_{3}$ is also $(1-3 \tau)$ in the configuration minimizing $\prod \alpha_{i}$. So we get that $\alpha_{1}+\alpha_{2}=$ $(2-4 \tau)-2(1-3 \tau)=2 \tau$. As each $\alpha$ is at most $\tau$, this forces $\alpha_{1}=\alpha_{2}=\tau$. 
It can be verified that all the constraints are satisfied, and so we get the required lower bound for $g(P)$ :

$$
g(P) \geq\left(\prod_{i} \alpha_{i}\right)\left(\frac{5 \tau-1}{\tau}\right) \geq \tau^{2}(1-3 \tau)^{2} \frac{5 \tau-1}{\tau} .
$$

We can now complete the proof of our main result.

Proof of Theorem 1.1 Wagner [8] proved that any point of depth $\tau n$ in $\mathbb{R}^{3}$ is contained in at least $f(\tau) n^{4}$ simplices spanned by $P$, where $f(\tau)=\left(4 \tau^{3}-6 \tau^{4}\right) / 4$ ! and $0.25 \leq$ $\tau \leq 0.5$. If $P$ has depth at least $\tau n$, where $\tau \geq 0.2889$, then as $f^{\prime}(\tau) \geq 0$ for $\tau \in$ $[0.25,0.5]$, we can deduce that $f(\tau) \geq f(0.2889)=0.00227$, and so there exists a point lying in at least $0.00227 n^{4}$ simplices spanned by $P$.

Otherwise, as depth is always an integer, $P$ has depth at most $\tau n-1$, where $\tau \leq 0.2889$. By Lemma 2.7, we can conclude that there exists a point lying in $g(\tau) n^{4}$ simplices, where $g(\tau)=\tau(1-3 \tau)^{2}(5 \tau-1)$. As $g^{\prime}(\tau) \leq 0$ for $\tau \in[0.25,0.3]$, we can deduce that $g(\tau) \geq g(0.2889)=0.00227$, and so there exists a point lying in at least $0.00227 n^{4}$ simplices spanned by $P$.

\section{Conclusion}

The conjecture of [6] that there always exists a point contained in at least $(n /$ $(d+1))^{d+1}$ simplices spanned by any $n$ points in $\mathbb{R}^{d}$ is an elegant one. So far, it has only been proven in $\mathbb{R}^{2}$ [4], and in this paper, we have made a step toward the optimal bound for $\mathbb{R}^{3}$. This indicates that the current lower bounds are not tight and gives more strength to their conjecture. The full conjecture, however, is still open.

Acknowledgements We thank the anonymous reviewers for valuable comments that improved both the content and style of this paper.

\section{References}

1. Alon, N., Kleitman, D.: Piercing convex sets and the Hadwiger-Debrunner $(p, q)$-problem. Adv. Math. 96(1), 103-112 (1992)

2. Alon, N., Bárány, I., Füredi, Z., Kleitman, D.J.: Point selections and weak e-nets for convex hulls. Comb. Probab. Comput. 1, 189-200 (1992)

3. Bárány, I.: A generalization of Caratheodory's theorem. Discrete Math. 40, 189-200 (1982)

4. Boros, E., Füredi, Z.: The number of triangles covering the center of an $n$-set. Geom. Dedic. 17(1), 69-77 (1984)

5. Bukh, B.: A point in many triangles. Electron. J. Comb. 13(10) (2006)

6. Bukh, B., Matoušek, M., Nivasch, G.: Stabbing simplices by points and flats. Discrete Comput. Geom. 43(2), 321-338 (2010). doi:10.1007/s00454-008-9124-4

7. Matoušek, J.: Lectures in Discrete Geometry. Springer, New York (2002)

8. Wagner, U.: On $k$-sets and applications. Ph.D. thesis, ETH Zurich, 2003 